\title{
Urgences
}

\section{Michel Savard, Le sourire des chefs, Montréal, Éditions du Noroît, 1987, 107 p. (avec sept dessins de Paul-Émile Saulnier).}

\section{Paul Chanel Malenfant}

Numéro 20, mai 1988

\section{Appellation contrôlée}

URI : https://id.erudit.org/iderudit/025486ar

DOI : https://doi.org/10.7202/025486ar

Aller au sommaire du numéro

\section{Éditeur(s)}

Urgences

\section{ISSN}

0226-9554 (imprimé)

1927-3924 (numérique)

Découvrir la revue

Citer ce compte rendu

Malenfant, P. C. (1988). Compte rendu de [Michel Savard, Le sourire des chefs, Montréal, Éditions du Noroît, 1987, 107 p. (avec sept dessins de Paul-Émile

Saulnier).] Urgences, (20), 71-74. https://doi.org/10.7202/025486ar d'utilisation que vous pouvez consulter en ligne.

https://apropos.erudit.org/fr/usagers/politique-dutilisation/ 


\section{MICHEL SAVARD: LE SOURIRE DES CHEFS, MONTRÉAL, EDITIONS DUNOROÎT, 1987, 107 p. (avec sept dessins de Paul-Émile Saulnier).}

Poésie de la concision minimaliste, de l'économie langagière, de la nomination photographique (en noir et blanc) des traits du réel: tels sont les invariants stylistiques qui caractérisent et unifient le dernier recueil de Michel Savard, Le sourire des chefs. Par ailleurs, les quatre suites qui constituent ce livre («petit déjeuner», «mur de briques/murale/(matériaux mixtes)», «cela» et "nationalismes») se présentent, du point de vue des motifs (ou matériaux) thématiques, comme des «objets» ou des «albums» autonomes, de telle sorte que leur disposition en quatuor relève, justement, d'une entreprise de "mixage ou d' «installation textuelle: le relief sémantique des éléments éclectiques rassemblés se trouve alors accusé par le caractère délibérément inusité de leur regroupement structural.

Avec la première partie, "petit déjeuner", le texte désigne, par l'instance titulaire, un commencement ou plutôt un «début» - déjà, il jette son «petit dé.... qui jamais n'abolira le hasard... - lequel se précisera, à l'incipit du poème initial, par l'évocation du "recommencement» du réveil et l'énonciation de la parole inaugurale: «chaque matin t'éveiller/c'est dires (p. 11).

À ce coup d'envoi succèdent des poèmes «matinaux* qui tour à toùr mettent en scène les gestes et objets usuels du «petit jour», l'instant aigu de la lame du rasoir dans la barbe, des alentilles de savon/à fleur d'oeils (p. 13), des «jus de fruits cigarettes et cafés» (p. 14), des arapports de circulation» (p. 16), des «croissants, la nappe, le beurre, le miel» (p. 18), bref tout un appareil du quotidien banal et routinier par lequel la poésie entend s'adonner à la prise photographique, comme en gros plans, de l'xin-signifiance» des données immédiates du réel. Mais qu'on ne s'y trompe pas: le sens du détail apparemment anodin ou la désignation laconique des choses n'induisent pas, chez Savard, le constat prosaïque d'une réalité êtale où le langage menacerait de se diluer; l'insistance du regard objectif (ou de l'objectif de la caméra) vise plutôt à une saisie de l'opacité parfois nauséeuse des choses, à la mise en évidence de leur «inquiétante (ou hallucinante) étrangeté», alors même que les choses, transmuées par les mots, deviennent d'autres choses, tels ce "gras reptile noir/le téléphone assis/(qui) soupire» (p. 19) ou encore cette absurde et magnifique "bête à bon Dieu/sur un parapluie rouge» (p. 20).

Dès lors, cette poésie, en donnant à «autrement» voir et à adifféremment» dire ce qui du monde pourrait paraître einsignifiant», parvient à l'efficace poétique de l'xinsensé»; plus encore, elle réalise, - à l'encontre du langage courant, langage d'usage et d'usure de ces achefs» qu' elle dénonce en la lettre même de son travail ou de ses stratégies, la remarquable adéquation entre la forme de ses dispositifs et le sens de son propos. Ainsi, pour dire la monotonie du «au jour le jour*, pour signifier la vacuité du *pareil au même», le texte engage à la fois la répétition sémantique et le mimétisme acoustique: 
ces «gestes à peine pris/dans l'écheveau du jour» (p. 12) convoqueront «les larves de faits de gestes/qui feront autre ce jour* (p. 14); «les journaux en liasses» violenteront le ajour naissant (p. 15), ce *maussade jour passablement/craméx (p. 22). Cette gestion de la reprise contrapuntique du «même» infère au poème une sorte de monochromie de la signification où le sens des choses se nivelle dans l'osmose des sons; c'est dans cette perspective que ces vers - «dans les logis l'eau/déroule sa fuite aux tuyaux/des bouil/loires baillent/des ustensiles cliquètent/des assiettes assiégées/ - induiront au constat $*$ (...) que la routine au trait prolongé/claquant des dents/conclut* ( $\mathrm{p}$. 23).

Tout se passe alors comme si la proximité du poème avec les substances et les sensations, proximité que supporte l'appellation des choses les plus immédiatement données au regard ou encore les plus banales, - et ainsi, en apparence, les moins poétiques - conduisait à une promiscuité, sémantique et acoustique, des mots les uns à l'égard des autres. De cette manière le «petit déjeuner convoque en sa suite, comme par décalque, tout un répertoire de apetits objets», dont la petitesse - de la même manière toute lettre est minuscule à l'attaque des poèmes - subrepticement se mue, en incisivité. Ainsi, «à la fenêtre l'oeil/scie ses faisceaux radar (p. 22), les «horloges (sont) pointeuses (p. 23), l'oeil se fait rasoir - kles yeux rasant le gris transvasex (p. 24). Enfin, ale café coupe/froid» (p. 17): on le voit, cette rêverie de l'incision et de la coupure gouverne jusqu'au découpage du vers qui constamment travaille la syncope, la rupture brusque et la cassure syntaxique: acertains font cent pas de ciment/puis les reprennent comme parole/trop vite donnée certains/vont vociférant et viennent» (p. 25). Ainsi, chez Savard, comme chez un Giguère par exemple, les vers sont bien des vers brisés.

Ces répercussions ou ces superpositions des vocables qui déterminent l'ubiquité sémantique, ces enchâssements, ces aclefs allitératives* ou ces écholalies qui traduisent une volonté d'amenuiser le sens, de le réduire à sa plus simple expression, bref de le porter à bout de souffle (de sons), se prolongent d'évidence dans la deuxième suite du recueil, intitulée «mur de briques/murales/(matériaux mixtes) : tant il est vrai que ce parti pris du mur sera aussi un parti pris du son, du mur du son. Voyons donc comment $\alpha[\ldots]$ catégorique/le mur de brique/fonce» (p. 31); comment aune brique (...) murit/dans le mur/son encastrement» (p. 35). Dans le grain du signifiant, ces vers réalisent ce qu'ils disent; à la lettre, ils encastrent les briques, ces mots qui élèvent le mur du poème (emmuré).

Pour traduire encore l'immuable opacité de ce mur qui, tel qu'en lui-même demeure, pour signifier la successivité à la fois étale et répétitive de son architecture, le poème se constitue en une sorte d'échafaudage réitératif, en une véritable architexture de la reprise analogique: «des mois déjà le mur/les briques qui font le mur/et le mur ses briques/nous arrêtent» (p. 38). Les énoncés évoluent par variations imperceptibles de sens, ils se métamorphosent, en sourdine, par similarités sonores; ainsi les vers afermement soutenue/par la fidélité du modeste mortier» génẻreront leurs analogons réversibles: «c'est dans les filets escaliers du mortier/qu'elle sinue» (p. 34). Ce 
"mixage* ou ce magnétisme systématique du son et du sens (ce «murmure* immobile du mur qui paradoxalement a[...] sait écouter/nos plus misérables paroles/sans leur prêter le moindre échov (p. 41)) s'est énoncé, "dès l'abord* de la suite qui désigne le mur, en «ce remuement d'air à peine» (p. 31) qui a suffi à son apparition. Depuis, par delà le mur, l'air (le son, le souffle) résonne de toutes parts, franchit le mur jusqu'en cette acaresse plus légère/plus délétère* (p. 33).

«au moins cela/ne perce pas/le mur des silences» (p. 63): à la concrêtion matérielle et sonore du mur, succède l'indétermination exacerbée de «cela», titre de la troisième partie du recueil (et la seule dont les textes (17), significativement, ne sont pas numérotés). Qu'est-ce, "cela»? Tour à tour la colère, la mort, la solitude, l'oppression de la police (contre la poésie: aau premier prétexte/cela réclame des papiers/viole des sceaux et dresse/des tables d'écoute» (p. 64)), la nostalgie du temps qui passe, l'insoutenable inflation des discours d'autorité de ces «inconnus aux visages tirés de circonstances/qui d'une voix d'outre-tombe dictent/l'essentiel des mesures incitatives propres à rétablir/l'ordre indispensable à l'exercice des libertés/fondamentales/les mesures l'ordre l'exercice» (p. 59). Cela c'est encore la dérisoire indifférentiation du sens: $\alpha$ cela/[...]/un char/pas un chat» (p. 62); c'est l'absurdité du mutisme de Dieu: «des semaines/qu'il pleut que Dieu/ne rêpond plus» (p. 69); c'est le manque de tout ce qui du réel se désiste, le défaut de la lettre perdue: «la nuit qui se referme/en claquant sur ses briques/où ne manquent au mot/que deux lett» (p. 69).

À la limite, "cela» c'est bien «le mur des silences» à l'envers du mur du son. Par une rare économie de moyens, Savard parvient ici à affirmer avec intensité — $-\alpha$ La redondance n' informe pas mais elle exprime», nous dit Jean Cohen - cette vacuité du sens et ce vertige de l'abîme (et de l'abyme) qui ressortent de l'exercice même du langage poétique, et qui, en un sens, lui confèrent sa fascinante existence. Il réalise à sa manière le projet de Flaubert "de faire un livre sur rien», inscrivant des textes qui sans cesse renvoient à eux-mêmes, disant "cela», cet indicible ou ce non-dit que dit la poésie.

«il y en a beaucoup d'autres/un mur au pays perdu/ne sait suffire/ mais ces regards toujours/reviennent se poser sur vous/quand le soir comme un vieux chien s'étend/et qu'il songe/à celaw (p. 73): cette strophe, clausule du dernier poème de la troisième partie du livre, effectue, par le recours au "mur» (de la deuxième suite), par la réfêrence terminale à l'indécidable «cela» (le "ça» pense, le "ça» parle... de la troisième suite) et par l'allusion au «pays perdu*, la jonction synthétique avec le quatrième volet du recueil: "nationalismes». Notons qu'en fin de parcours les postures "matinales» du "petit déjeuner» (première suite) et de la "petite» histoire personnelle accèdent aux incidences "nationales» et collectives de l'Histoire: "ainsi nous sommes/ Québécois malgré qu'il nous en coûte» (p. 79). À partir de ce leitmotiv anaphorique (uainsi nous sommes»), le poème tend à l'incantation parodique (et pathétique) de notre légendaire absence au monde, de notre résignation béate à l'inexistence. Par l'impact de telles images, la trépidation syncopée des rythmes, la virulence du propos «nationaliste», on pourrait, à l'occasion, 
songer à Miron ou au Michel Beaulieu du Cercle de justice et de L'octobre suivi de Dérives. Mais est-ce l'actualité politique retrouvée de ces éclats de voix? ou le clinquant passéiste du complexe de notre spetite noirceur»? Devant tout ce folklore de l'aliénation délirante repris par Savard, le lecteur se demande si la nostalgie est bien toujours ce qu'elle était: «ainsi nous sommes/à mi-chemin de rien du tout/dans la dérision de nos reels/de tourtières et de bouts de gras/sautillant step by step les uns/à bonne portée de l'enfer/des autres* (p. 102). S'ajoutent à ces humeurs tel effet de mémoire («nous observons en pleine absence/s'effacer Nelligan l'enfance/de nos écrans l'amour tu* (p. 87)); la kyrielle de nos signes distinctifs, la «broche à foin* (p. 91), *la bavette de nos dires» (p. 90), *nos lainages de résistance» (p. 93), *nos langes nos lainages nos flanelles nous/nos linceuls souillés de silence* (p. 99) et notre ajoie de giguer imaginez/envoléex (p. 97); et jusqu'à cette truculence *bien-de-chez-nous-dont-on-se-moque-joyeux-content*: aclaques dans les flaques paradons/la bouteille à la main les autorités/ferment les rues et certains yeux/les putes sont sorties/voir passer les caves/ (p. 88)!!! Certes la dérision et l'ironie sont thérapeutiques...

Il reste que sans ces déplorables petites inorales et ces cartes de parti, Michel Savard est un très solide poète, au métier parfaitement éprouvé. Peut-être le dernier acte du Sourire des chefs est-il tout simplement un acte manqué? Il reste aussi que je viens de relire ce qu'écrit Breton, dans Arcane 17 , à propos des «nationalismes», justement.

Paul Chanel Malenfant 